\title{
ARTICLE
}

\section{Macrophage-mediated chronic lymphocytic leukemia cell survival is independent of APRIL signaling}

\author{
MHA van Attekum ${ }^{1,2}$, S Terpstra ${ }^{1,2}$, E Reinen ${ }^{1,2}$, AP Kater $^{1,3,4}$ and E Eldering ${ }^{2,3,4}$
}

Survival of chronic lymphocytic leukemia (CLL) cells is mainly driven by interactions within the lymph node (LN) microenvironment with bystander cells such as T cells or cells from the monocytic lineage. Although the survival effect by T cells is largely governed by the TNFR ligand family member CD40L, the exact mechanism of monocyte-derived cell-induced survival is not known. An important role has been attributed to the TNFR ligand, a proliferation-inducing ligand (APRIL), although the exact mechanism remained unclear. Since we detected that APRIL was expressed by CD68+ cells in CLL LN, we addressed its relevance in various aspects of CLL biology, using a novel APRIL overexpressing co-culture system, recombinant APRIL, and APRIL reporter cells. Unexpectedly, we found, that in these various systems, APRIL had no effect on survival of CLL cells, and activation of NF- $\kappa$ B was not enhanced on APRIL stimulation. Moreover, APRIL stity mulation did not affect CLL proliferation, neither as single stimulus nor in combination with known CLL proliferation stimuli. Furthermore, the survival effect conveyed by macrophages to CLL cells was not affected by transmembrane activator and CAML interactor-Fc, an APRIL decoy receptor. We conclude that the direct role ascribed to APRIL in CLL cell survival might be overestimated due to application of supraphysiological levels of recombinant APRIL.

Cell Death Discovery (2016) 2, 16020; doi:10.1038/cddiscovery.2016.20; published online 21 March 2016

\section{INTRODUCTION}

Interactions of chronic lymphocytic leukemia (CLL) cells with bystander cells in tumor microenvironments, such as the lymph node (LN), provide them with essential survival signals. Upregulation of pro-survival B-Cell Lymphoma-2 family members occurs on stimulation with $T$ cells or with monocyte-derived cells such as macrophages or nurse-like cells (NLCs). ${ }^{1}$ Although the tumor necrosis factor (TNF) receptor ligand family member CD40L can account largely for the survival effect by $T$ cells, ${ }^{2}$ several factors have been described to have a role in the CLL cell survival effect governed by monocyte-derived cells. ${ }^{3,4}$ A prominent factor in this context is the TNF family member a proliferation-inducing ligand (APRIL). ${ }^{4}$

Under physiological conditions, APRIL has diverse roles in the development of B cells. It binds to its cognate receptors transmembrane activator and CAML interactor (TACI) and B-cell maturation antigen (BCMA) after which TNF receptor associated factors are recruited to the receptor that relay the signal intracellularly. APRIL has furthermore been shown to signal via binding to heparan sulfate proteoglycans on the cell surface of its target cell. ${ }^{5}$ In healthy B cells, APRIL signaling has a role in the induction of CD40L-independent class-switch recombination, ${ }^{6}$ proliferation, ${ }^{7}$ and sustained survival of plasmablasts. ${ }^{8}$ APRIL has been reported to be expressed by several cell types including macrophages, ${ }^{9}$ stromal cells, ${ }^{10}$ CLL cells, ${ }^{11}$ and NLCs, ${ }^{12}$ which are CLL cell-differentiated monocytes that have been shown to induce survival of CLL cells. ${ }^{3}$ APRIL is produced as either a membrane bound or soluble factor, depending on which alternative transcript(s) is/are expressed by the cell. ${ }^{13}$ Furthermore, APRIL can be synthesized as part of a hybrid transcript called TWEPRIL (TWEAK-APRIL) together with TNF-related weak inducer of apoptosis (TWEAK), after which it is anchored to the cell membrane by virtue of the TWEAK domain. ${ }^{14}$ Both TWEPRIL and the secreted alpha transcript variant of APRIL can be cleaved by furin in the Golgi apparatus or at the cell membrane, respectively. ${ }^{15}$ In contrast, the membrane-bound delta variant lacks the furin cleavage domain. ${ }^{13}$

In its ability to support cells, APRIL contributes to the growth of several malignancies, ${ }^{16}$ and serum APRIL levels are correlated with worse prognosis, ${ }^{17}$ which was also shown for CLL. ${ }^{18}$ Furthermore, APRIL overexpression by transgenesis in the T-cell leukemia/ lymphoma $1 \mathrm{~A}(\mathrm{TCl}-1) \mathrm{CLL}$ mouse model is associated with enhanced disease severity ${ }^{19}$ and APRIL transgenic mice show an enhanced proliferation of peritoneal B-1 cells, ${ }^{20}$ which are considered to be the precursor cells for CLL in mice. ${ }^{21}$ These effects are thought to result from induction of CLL cell survival by APRIL via activation of nuclear factor kappa-light-chain-enhancer of activated $B$ cells $(\mathrm{NF}-\kappa \mathrm{B}){ }^{4,12}$ Altogether, these data suggest a role for APRIL in CLL biology. These findings have, however, been questioned by other reports, in which no survival effect on CLL cells was found. ${ }^{22,23}$

To mechanistically dissect the role of APRIL, we used several complementary approaches to study its effects on CLL survival, activation, proliferation, and to investigate its role in macrophage-mediated survival. Surprisingly, we could not detect a direct effect of APRIL on CLL cells. Furthermore, although macrophages induce CLL survival, this effect appears to be independent of APRIL.

\footnotetext{
${ }^{1}$ Academic Medical Center, Department of Hematology, University of Amsterdam, Meibergdreef 9, 1105 AZ, Amsterdam, The Netherlands; ${ }^{2}$ Academic Medical Center,

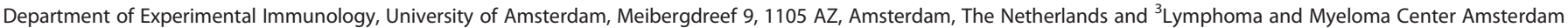
(LYMMCARE), Meibergdreef 9, 1105 AZ, Amsterdam, The Netherlands.

Correspondence: E Eldering (e.eldering@amc.nl)

${ }^{4}$ Shared senior authorship.

Received 18 January 2016; accepted 3 February 2016; Edited by R Aqeilan
} 


\section{RESULTS}

APRIL is expressed by macrophages in the CLL LN and CLL cells express APRIL receptors

We first addressed whether APRIL is expressed in the CLL LN by performing $\mathrm{qPCR}$ on total RNA lysates from CLL LNs. These results show that APRIL expression in CLL LNs was $\sim 4$ times higher compared with a control systemic lupus erythematosus (SLE) LN extract. As negative control, NIH-3T3 mouse embryofibroblasts (3T3) had no APRIL expression (Figure 1a).

Next, we verified this finding on protein level using immunohistochemistry by staining for APRIL and macrophage marker CD68. As APRIL has been described to induce cell proliferation, ${ }^{7}$ we also stained for proliferation marker Ki67. APRIL was expressed by the large majority of CD68+ cells in both CLL and SLE LNs, but there was no spatial association with Ki67+ lymphocytes in the CLL LNs (CLL LN, Figure 1b and SLE LN, Supplementary Figure S1). Furthermore, expression of APRIL receptors BCMA and TACI was clearly detectable on CLL cells isolated from peripheral blood (PB; Figure 1c).

In summary, APRIL is expressed in the CLL LN by macrophages and APRIL receptors are present on CLL cells.

No survival effect on CLL cells by in vitro APRIL stimulation To explore direct functional effects of APRIL on CLL cells, we transduced NIH-3T3 cells (DSMZ, Braunschweig, Germany) with three different membrane-docked APRIL constructs (Figure 2a). We thus generated a system similar to the widely used TNF family member CD40L overexpressing NIH-3T3 line (3T40), ${ }^{24-26}$ thereby ensuring trimerization of APRIL and expression on the cell membrane. The first cell-line expresses the membrane-bound TWEPRIL hybrid mRNA, with mutated furin consensus sites to render it uncleavable (3TA). In the second and third constructs (3T4A and 3T4sA), the intracellular and transmembrane regions of CD40L were fused to the extracellular domain of APRIL, without or with an interposed spacer ('s') region. The $3 T 40$ cell line ${ }^{24-26}$ was used as a control.

APRIL expression in these cell lines was then verified by qPCR (Figure 2b) and western blot (Figure 2c), and signaling competence was tested using Jurkat-TACl:FAS (JTF) reporter cells $^{27}$ (Figure 2d). These JTF cells undergo apoptosis on TACI signaling as a result of intracellular FAS domains, and provide a sensitive read-out for APRIL binding to its cognate receptor (Figure 2a). Conditioned medium from APRIL overexpressing HEK293T cells (rhA med) and recombinant human APRIL (data not shown) were included as controls (Figure 2d). These data showed that all cell lines from our in vitro co-culture system express APRIL and that the expressed APRIL is able to signal via $\mathrm{TACl}$.

These APRIL expressing $3 T 3$ cells were subsequently used to test whether APRIL induced CLL cell survival. In contrast to 3T40 cells, we found no survival effect by any of the APRIL constructs or by rhA after $72 \mathrm{~h}$ co-culture (Figure 2e). Similarly, we could not detect a survival effect of conditioned supernatant from APRIL transfected HEK293T cells compared with supernatant from mock
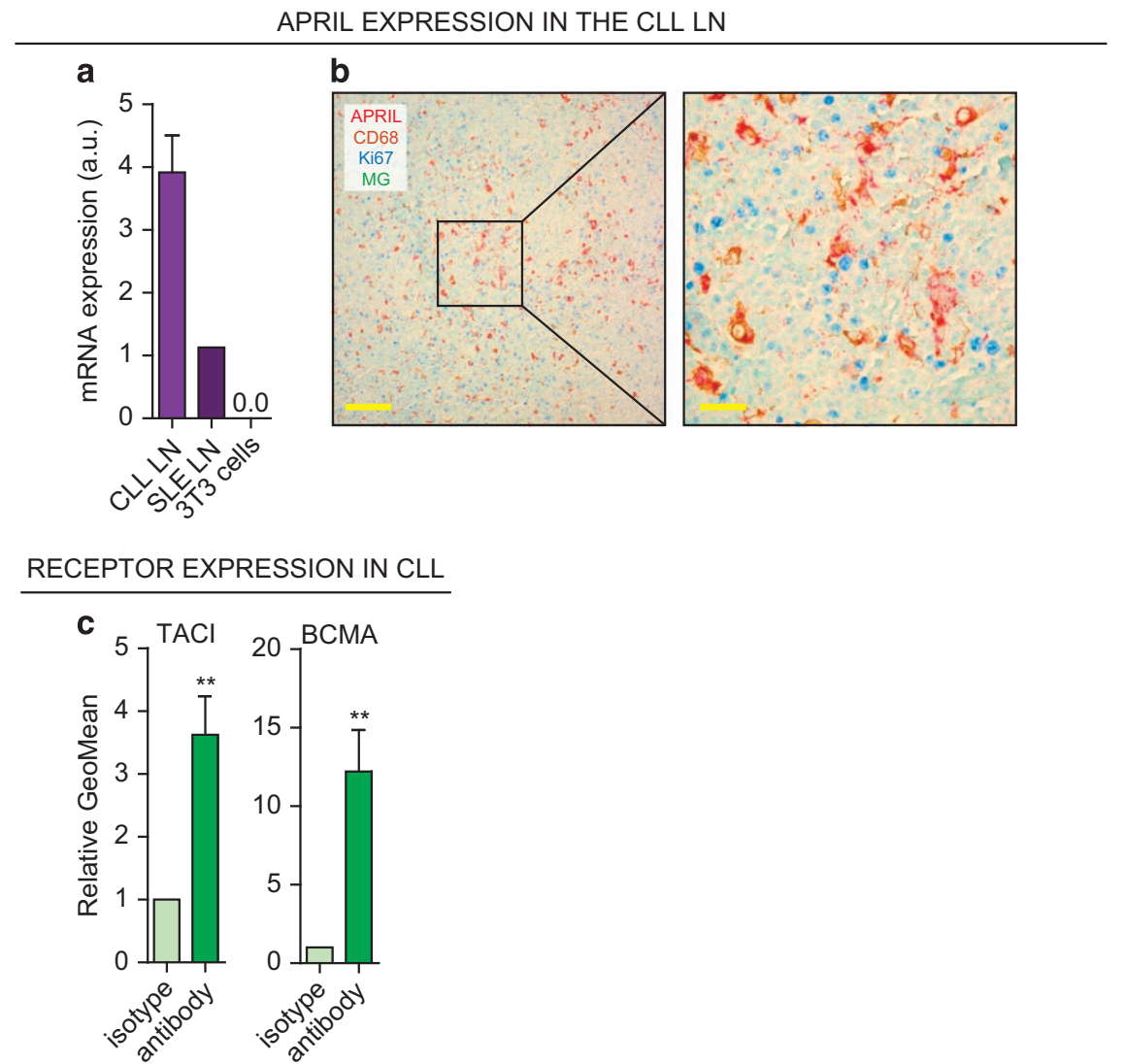

Figure 1. APRIL is present in the CLL LN and CLL cells express APRIL receptors. (a) After total RNA lysis of paraffin-embedded LN material or control NIH-3T3 (3T3) cells, APRIL mRNA levels were determined by performing a qPCR on CLL LN material $(N=3)$ and an SLE LN as positive and 3 T3 cells as negative control. All qPCRs were performed in triplo. a.u. denotes arbitrary units. (b) Paraffin embedded LN slides from six CLL patients were immunohistochemically stained for APRIL, macrophage marker CD68, proliferation marker Ki67, and nuclear counterstain methyl green (MG). Data shown are representative of $N=6$. Scale bar represents $200 \mu \mathrm{m}$ (left) or $50 \mu \mathrm{m}$ (right). (c) CLL cells ( $N=6)$ isolated from PB were stained for APRIL receptors TACI and BCMA or with the relevant isotype controls and analyzed by flow cytometry. Bars show mean \pm S.E.M. ${ }^{* *} P<0.01$ in a paired $t$-test. 
IN VITRO APRIL SYSTEM
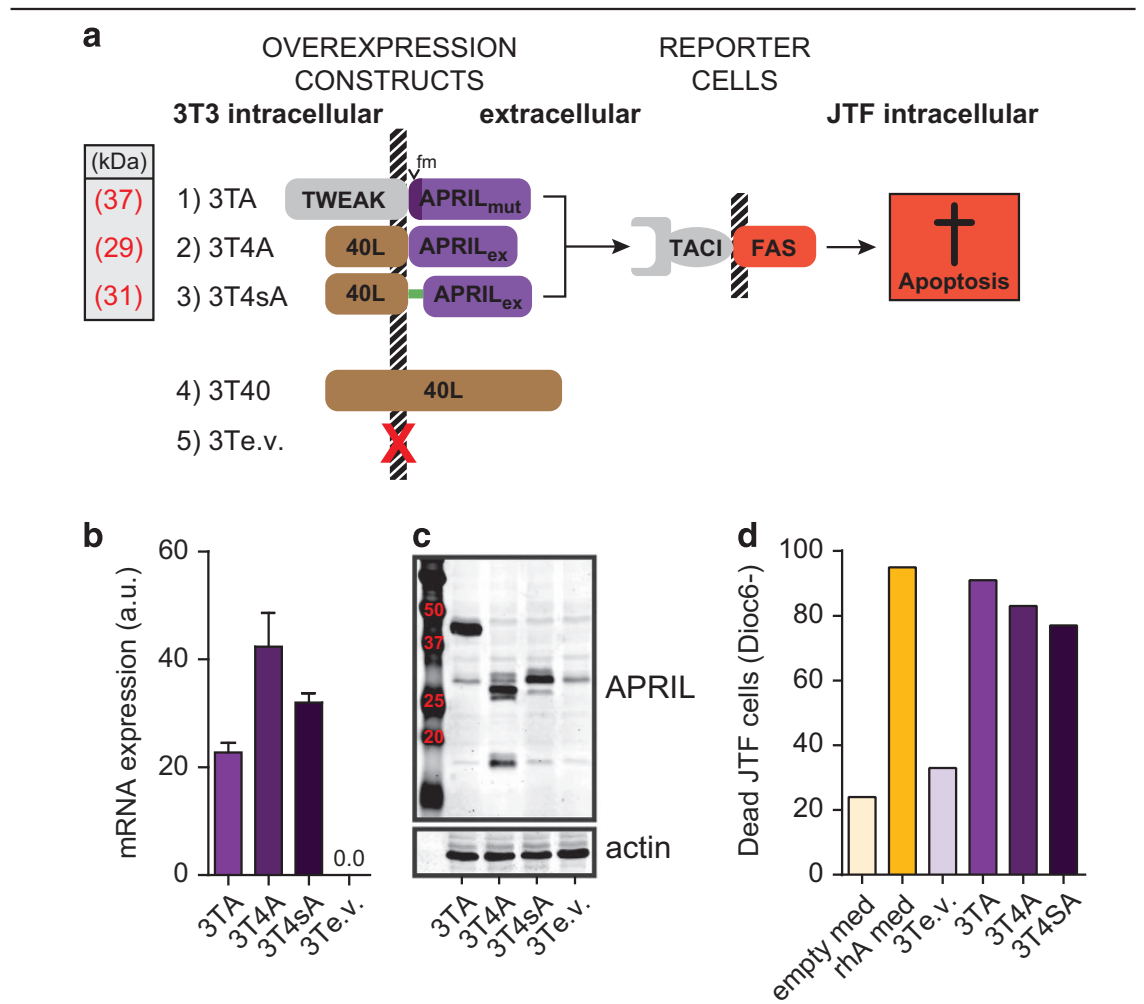

\section{CLL SURVIVAL}

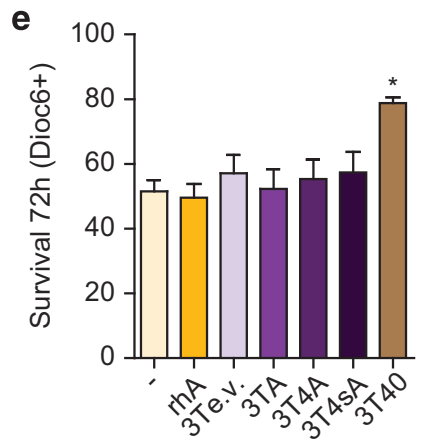

Figure 2. APRIL does not induce CLL cell survival. (a) Depiction of APRIL overexpressing cell lines, control cell lines, and reporter cells used in co-culture experiments. NIH-3T3 cell lines overexpressing three different membrane-bound APRIL constructs were created (Methods section). Apoptosis in APRIL reporter JTF cells is induced on APRIL signaling, as TACI signaling triggers the FAS cell-death pathway. Full-length CD40L overexpressing $3 \mathrm{~T} 3$ cells (3T40) and empty-vector transduced 3T3 cells (3Te.v.) are used as controls. Mutated furin sites are indicated by 'fm', the spacer region is depicted by a green line. All constructs are drawn to scale. (b) APRIL mRNA expression levels of the different APRIL overexpressing cell lines were tested by qPCR and compared with cells overexpressing 3Te.v. The qPCR was performed in triplo and bars show mean \pm S.E.M., a.u. denotes arbitrary units. (c) APRIL protein expression levels of the different APRIL overexpressing cell lines were tested by western blot and compared with cells overexpressing 3Te.v. The predicted molecular weights of the APRIL fusion proteins are indicated in Figure 2a. (d) Cell lines described in Figure $2 a$ were seeded as feeder layers, and JTF reporter cells ${ }^{27}$ were plated on top. Concurrently, JTF reporter cells were cultured in conditioned medium from APRIL (rhA med) or mock (empty med) transfected HEK293T cells. After 24 h coculture, the percentage of dead (Dioc6 negative) JTF reporter cells was determined by Dioc6-PI staining. (e) CLL cells were cultured for $72 \mathrm{~h}$ without stimulation (-) or with $200 \mathrm{ng} / \mathrm{ml}$ rhA. Likewise, CLL cells were co-cultured on the indicated APRIL expressing or control cell lines. Next, survival was determined by Dioc6-PI staining. Viable cells were defined as Dioc6-positive cells. CD40L overexpressing feeder cells (3T40) were used as a positive control for CLL cell survival. Bars show mean \pm S.E.M. for $N \geqslant 8$ for each condition. ${ }^{*} P<0.05$ in a ANOVA with Tukey post hoc tests. When testing for significant differences, rhA was compared with unstimulated cells and 3T3 overexpression cell lines to 3Te.v.

transfected cells (data not shown and Supplementary Figure S2). Using the same APRIL stimuli, survival of CLL cells was measured at later time points (3, 6 and 10 days). In accordance with the results obtained at $T=72 \mathrm{~h}$, APRIL did not significantly increase CLL survival, although a minor effect could be observed at day 10 for rhA (Supplementary Figure S2), as reported before. ${ }^{28}$
No NF- $\kappa$ B activation, activation marker expression, or cell division in CLL cells exposed to APRIL

As several TNF family members can induce $N F-\kappa B_{1}{ }^{29}$ we investigated if APRIL is able to induce NF- $K B$ activation by performing an NF- $K B$ DNA-binding enzyme-linked immunosorbent assay and found that, as expected, 3T40 cells induced both the 

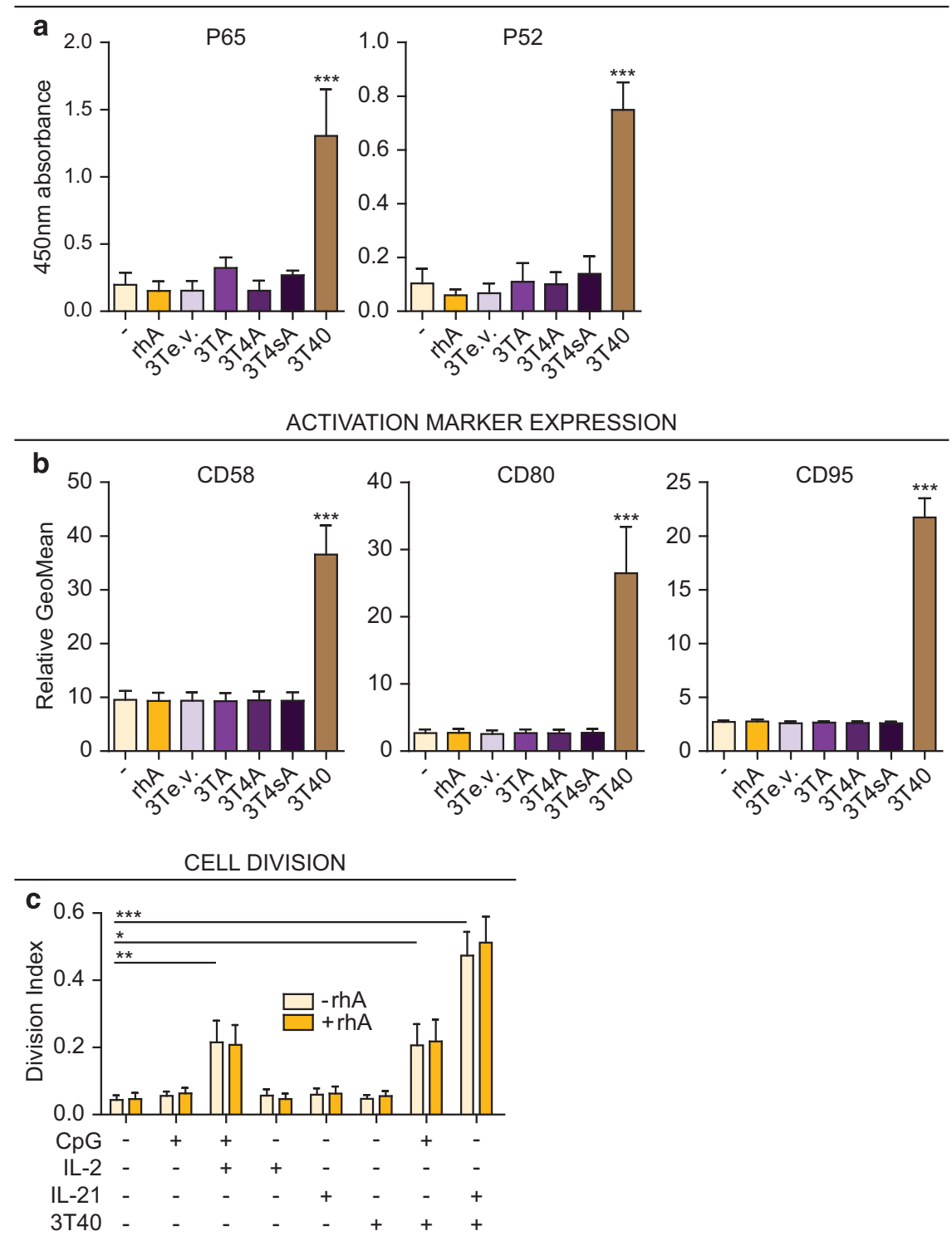

Figure 3. APRIL does not induce NF- $k B$ signaling, activation marker expression, or cell division in CLL cells. (a) CLL cells were cultured as in Figure $2 \mathrm{e}$ and nuclear extracts were prepared after $24 \mathrm{~h}$. The binding of activated canonical p65 and non-canonical p52 NF-KB subunits to consensus sequence oligonucleotides was then determined using enzyme-linked immunosorbent assay (ELISA). CD40L overexpressing feeder cells (3T40) were used as a positive control for NF-KB activation. Bars show mean \pm S.E.M. for $N=3$ for $3 T A$ and $3 T 4 s A$ and $N=5$ for the other conditions, respectively. ${ }^{* * *} P<0.001$ in an ANOVA test with Tukey post hoc analysis. When testing for significant differences, rhA was compared with unstimulated cells and 3T3 overexpression cell lines to empty-vector transduced 3T3 cells (3Te.v.). (b) CLL cells were cultured as in Figure 2e for $72 \mathrm{~h}$ and expression levels of activation markers CD58, CD80 and of CD95 were determined using flow cytometry. CD40L overexpressing feeder cells (3T40) were used as a positive control for activation marker induction. Bars show mean \pm S.E.M. for $N=3$. ${ }^{* * *} P<0.001$ in an ANOVA test for repeated measures with Tukey post hoc analysis. When testing for significant differences, rhA was compared with unstimulated cells and 3T3 overexpression cell lines to 3Te.v. (c) CFSE-stained CLL cells were cultured with various stimulations as indicated and as described, ${ }^{2}$ each time with or without rhA. After 4 days, the CFSE dilution was visualized by flow cytometry and division indices were calculated. Bars show mean \pm S.E.M. for $N=6$. ${ }^{*} P<0.05 ;{ }^{*} P<0.01 ;{ }^{* *} P<0.001$; Each stimulation without rhA (light colored bars) was compared with the unstimulated condition in an ANOVA test for repeated measures with Dunnet's post hoc analysis and the differences between - and +rhA for each condition were determined using a two-way ANOVA with Bonferroni correction.

canonical (p65) and non-canonical (p52) pathway in CLL cells. In contrast, no NF- $\kappa B$ activation could be detected after stimulation with various APRIL constructs or rhA (Figure 3a), and known NF-KB target transcripts were not induced (data not shown).

Strong CD40 stimulation via cell-bound CD40L induces highlevel NF-KB activation in CLL cells. We have previously found that a CD40 stimulating antibody that induces moderate stimulation is able to upregulate activation markers CD58, CD80 and also CD95 (data not shown), indicating a higher sensitivity of this read-out.
We therefore tested the potential of APRIL in this context, but in contrast to CD40L stimulation, APRIL stimulations did not upregulate the indicated markers (Figure $3 \mathrm{~b}$ ).

To study APRIL's potential involvement in CLL cell proliferation, ${ }^{16}$ cell division was traced using carboxyfluorescein succinimidyl ester (CFSE) labeling, and the division index was calculated after various proliferation stimuli in the presence or absence of rhA. In line with a previous report, ${ }^{2}$ we found an increased proliferation of CLL cells after stimulation with CpG 
+interleukin (IL)2, 3T40+CpG, and 3T40+IL21, but no effect of rhA either as a single agent or in combination with these stimuli (Figure 3c).

Summarizing, although we found that CD40L is able to induce NF- $\kappa$ B activation in CLL cells, activation marker expression and cell proliferation in combination with CpG or IL21, similar effects were not detectable after APRIL stimulation.

Macrophage-mediated CLL survival is independent of APRIL $\mathrm{We}^{30}$ and others ${ }^{3}$ have previously found that monocyte-derived cells such as macrophages are able to induce survival of CLL cells, and it was suggested that survival by monocyte-derived cells is dependent on APRIL. ${ }^{12}$ Although we did not observe a survival effect of stimulation with APRIL as a single stimulus (Figure 2e), the effects of APRIL could be dependent on other macrophageexpressed cytokines.

We, therefore, first generated M1 macrophages in vitro by differentiating healthy donor-isolated monocytes with interferon gamma (IFN-Y; R\&D systems, Minneapolis, MN, USA). We then tested whether APRIL was expressed by these macrophages on western blot and found high expression in differentiated macrophages compared with low expression in monocytes and no expression in control 3T3 cells (Figure 4a inset and Supplementary Figure S3). The APRIL signaling capacity of these macrophages was then tested by comparing cell-death induced by macrophages in JTF reporter cells with the JTF death-to-rhA dose-response curve. The APRIL signaling capacity of macrophages was between that of 0 and $3.13 \mathrm{ng} / \mathrm{ml} \mathrm{rhA}$ (Figure 4a).

To inhibit potential APRIL signaling during macrophage stimulation, we used TACl-Fc (R\&D systems), a chimeric decoy receptor for APRIL. ${ }^{31}$ We tested the activity of TACl-Fc by its ability to inhibit macrophage-induced cell death of JTF reporter cells cultured on macrophages. We found that TACI-Fc dose-dependently reduced APRIL signaling from macrophages (Figure 4b).

We then cultured CLL cells on macrophages and measured CLL survival in the absence or presence of $2.5 \mu \mathrm{g} / \mathrm{ml} \mathrm{TACl-Fc}$, the concentration at which macrophage-induced APRIL signaling was completely reverted. These data show that abrogation of APRIL signaling did not decrease the survival effect conveyed by macrophages (Figure 4c), suggesting no direct role for APRIL in macrophage-mediated CLL survival. Similarly, when culturing CLL cells on NLCs ${ }^{12}$ generated by 10 days stimulation of monocytes with CLL cells, inhibition of APRIL signaling by TACI-FC did not affect CLL survival (Figure 4d).

\section{DISCUSSION}

We studied potential effects of TNF-family member APRIL on CLL cells, using complementary approaches and JTF reporter cells to verify the functionality of recombinantly expressed APRIL and the TACl-Fc decoy receptor. In contrast to our initial expectations, we could not detect an effect of APRIL on either CLL cell survival, cell activation, NF- $K B$ activation or cell proliferation. In addition, we could not detect a direct role of APRIL in macrophage-mediated CLL cell survival.

Various studies reported on the effects of APRIL on CLL. Although some publications show an increased in vitro survival of CLL cells by rhA when used at a concentration of $500 \mathrm{ng} / \mathrm{ml}$, ${ }^{4,12}$ our experiments using $200 \mathrm{ng} / \mathrm{ml}$ rhA (Figure 2e) are in line with the data of several other groups that were unable to find effects of recombinant APRIL, either alone ${ }^{22}$ or in combination with B-cell activating factor and chemokine (C-X-C motif) ligand 1 (CXCL) $12 .{ }^{23}$ Also, we established that the amount of APRIL produced by macrophages is $>100$ orders of magnitude lower compared with concentrations used in the reports that detect survival by APRIL. Although APRIL may induce survival at high concentrations, ${ }^{4,12}$ this effect might be supraphysiological. Furthermore, concerning
APRIL IN MACROPHAGE-MEDIATED CLL SURVIVAL
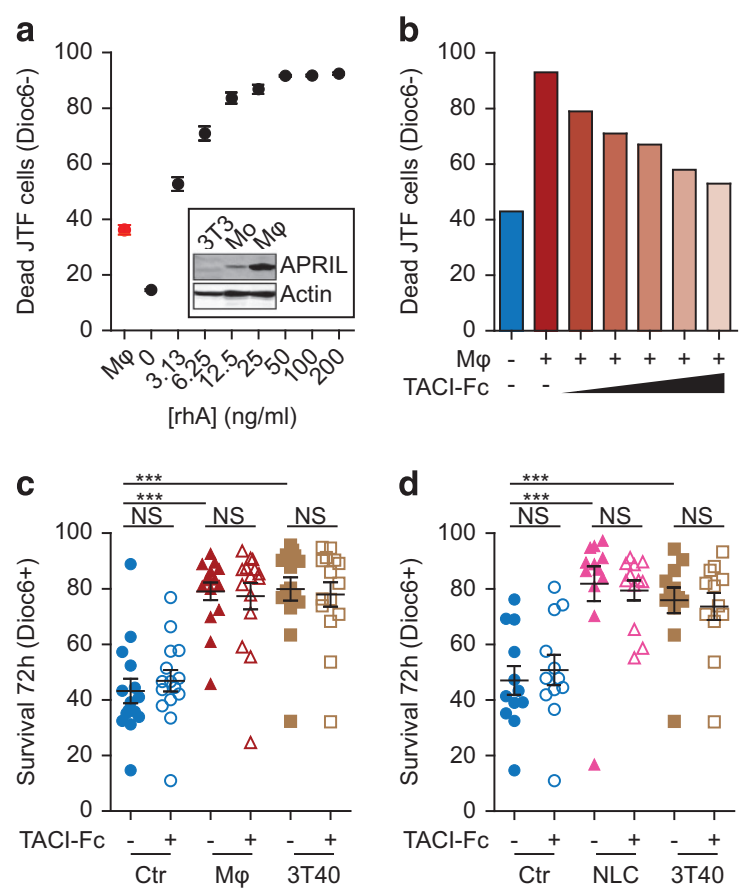

Figure 4. APRIL is expressed by macrophages, but has no role in macrophage-mediated survival. (a) JTF reporter cells were stimulated for $24 \mathrm{~h}$ with different concentrations of rhA or with M1-differentiated macrophages. Consequently, cell viability was determined as in Figure $2 \mathrm{~d}$ and the macrophage-induced cell death was plotted alongside of the rhA titration curve. All conditions were performed in triplo and mean \pm S.E.M. are shown. Inset: APRIL expression was determined in these macrophages $(M \varphi)$ by western blot and compared with monocytes (Mo) and untransduced 3T3 cells as negative control. (b) JTF reporter cells were stimulated with M1-differentiated macrophages as in Figure $4 a$ in the presence of increasing concentration of the APRIL decoy receptor TACI-Fc (from $0.25 \mu \mathrm{g} / \mathrm{ml}$ to $2.5 \mu \mathrm{g} / \mathrm{ml}$ ) or control IgG after which cell viability of the JTF cells was measured as in Figure $2 \mathrm{~d}$. (c) Confluent feeder layers of macrophages $(M \varphi)$ were generated as in Figure $4 a$ and 3T40 feeder layers as in Figure 2e. These feeder layers or empty wells (Ctr) were then pre-incubated for $30 \mathrm{~min}$ with TACI-Fc to suppress APRIL signaling or control IgG after which CLL cells were added on these feeder layers and co-cultured for $72 \mathrm{~h}$. Next, survival of the CLL cells was determined as in Figure 2e. Each point is one CLL sample $(N=15)$ cultured in the indicated condition and mean \pm S.E.M. are indicated. ${ }^{* * *} P<0.001$; NS, not significant, in an ANOVA test for repeated measures with Dunnett's post hoc analysis. (d) Confluent feeder layers of NLCs were generated by differentiating monocytes for 10 days using CLL cells. After washing, their survival effect on CLL cells in the presence of absence of TACl-Fc was determined as in Figure 4c. Each point is one CLL sample $(N=12)$ cultured in the indicated condition and mean \pm S.E.M. are indicated. ${ }^{* *} P<0.001$; NS, not significant, in an ANOVA test for repeated measures with Dunnett's post hoc analysis.

the survival effect of APRIL on non-malignant B cells, several groups have shown that APRIL is also dispensable in this context. $^{32}$

In the TCl-1 mouse model for CLL, we found that overexpression of human APRIL results in enhanced disease progression and shorter survival. ${ }^{19}$ In light of these results, our current in vitro findings were also unexpected. In the APRIL overexpressing Tcl-1 model, the construct encoding human APRIL is under control of the Lck promoter. APRIL is thus predominantly expressed by T cells, and is present in the serum at a concentration comparable to our in vitro systems (data not shown). As T cells not only interact with other lymphoid cells including (leukemic) B cells but also with 
myeloid-derived immune cells, it cannot be ruled out that the observed effects occur indirectly via other cells in the tumor microenvironment. Theoretically, the differences could also be due to distinct APRIL effects in the mouse compared with human situation.

We found no evidence that CLL proliferation is enhanced either in vitro or ex vivo by APRIL. These data are in line with another publication in which no significant proliferative effect of APRIL medium in the presence of CpG was found. ${ }^{28}$ Studies on the effects of APRIL on proliferation of healthy $B$ cells have been inconclusive; APRIL knockout mice for instance show normal B-cell proliferation in vitro ${ }^{33}$ and mice deficient for the APRIL receptor $\mathrm{TACl}$ paradoxically show increased B-cell proliferation, ${ }^{34}$ whereas BCMA knockout mice show no overt phenotype. ${ }^{35}$

In conclusion, our data indicate that APRIL does not directly mediate survival and proliferation of CLL cells. Consequently, APRIL signaling as therapeutic target in CLL might be beneficial in consideration that potential effects might be indirect.

\section{MATERIALS AND METHODS}

Patient samples

Patient material was obtained from CLL patients, after written informed consent and approval by our Ethical Review Board in agreement with the Helsinki Declaration of 1975, revised in 1983, as described before. ${ }^{36}$ All samples contained at least $90 \% \mathrm{CD}^{+} / \mathrm{CD} 19^{+}$cells (Supplementary Table S1). In all experiments, CLL cells were used at a final concentration of $1.5 \times 10^{6} \mathrm{cells} / \mathrm{ml}$.

\section{APRIL overexpression cell lines and other APRIL stimulations}

Mouse embryofibroblasts $\mathrm{NIH}-3 \mathrm{~T} 3$ cells were transduced for stable overexpression with pBABE vectors expressing (1) TWEPRIL (NM_172089.3) with mutated furin cleavage sites $(92 R R \rightarrow A A$ and $104 R R \rightarrow A A)$, (2) the transmembrane domain of CD40L (amino acids 1-112 of NM_000074.2) fused to the extracellular part of APRIL (amino acids 105-252 of NM_003808.3) without a linker region, or (3) with a linker region (PAAAAAASAAAAAAWVPVAT; Figure 2a), (4) $\mathrm{CD} 4 \mathrm{~L}^{2}{ }^{37}$ or (5) empty vector, and all transduced cells were selected using puromycin. All constructs were sequence verified before transduction. Cells were cultured in IMDM supplemented with (IMDM+/+): $10 \%$ fetal bovine serum (FBS, Invitrogen, Carlsbad, CA, USA), $100 \mathrm{u} / \mathrm{ml}$ Penicillin $-100 \mu \mathrm{g} / \mathrm{ml}$ Streptomycin (Life Technologies, Austin, TX, USA), 2 mM L-glutamine (Life Technologies) and $0.00036 \% \beta$-mercaptoethanol (Sigma, St. Louis, MO, USA). When used as adherent feeder layer, fibroblasts were irradiated ( $30 \mathrm{~Gy}$ ) to stop proliferation before being seeded. After feeder cell adhering, CLL cells were plated on the respective cells. Where indicated $(\mathrm{rhA}) 200 \mathrm{ng} / \mathrm{ml} \mathrm{rhA}$ was added to the culture medium, or culture medium conditioned on rhAoverexpressing HEK293T cells was added to the CLL cells at $80 \%$ final volume (rhA med).

\section{Immunohistochemistry}

Paraffin embedded CLL LN tissue was obtained from our institute's pathology department. Four-micron sections were de-waxed by immersion in xylene and hydrated by serial immersion in ethanol and TBS. Antigen retrieval was performed by heating sections for $20 \mathrm{~min}$ in sodium citrate buffer $(10 \mathrm{mM}$ sodium citrate, $0.05 \%$ Tween $20, \mathrm{pH} 6.0)$. Sections were washed with TBS $(2 \times 10 \mathrm{~min})$ and blocked with Ultra V block (Thermo Scientific, Waltham, MA, USA) for $10 \mathrm{~min}$ at $\mathrm{T}_{\text {room }}$. Sections were incubated with primary antibody APRIL-y2 (1:1000 Enzo LifeSciences, Farmingdale, NY, USA) in normal antibody diluent (ImmunoLogic, Duiven, The Netherlands) overnight at $4{ }^{\circ} \mathrm{C}$. After washing, sections were incubated with postantibody block (ImmunoLogic) and subsequently incubated with secondary Polymer a-Rb/AP antibody (ImmunoLogic) followed by visualization by Vector Red (Vector Laboratories, Burlingame, CA, USA). A second antigen retrieval was performed for $10 \mathrm{~min}$ at $98^{\circ} \mathrm{C}$ in TRIS-EDTA $(\mathrm{pH}=9.0)$ and after Ultra $V$ block (Thermo Scientific), sections were incubated with a combination of primary antibodies directed against CD68 (PG-M1, Dako, Carpinteria, CA) and Ki67 (SP6, Klinipath, Duiven, The Netherlands), both 1:2000 in normal antibody diluent for $1 \mathrm{~h}$ at $\mathrm{T}_{\text {room. }}$. After washing, a combination of Polymer a-Rb/AP and Polymer a-Ms/HRP (both Immunologic) was added for $30 \mathrm{~min}$ at $\mathrm{T}_{\text {room }}$ and antibody binding was visualized by Vector Blue (Vector Laboratories) and subsequent DAB (ImmunoLogic) staining after which slides were counterstained with methyl green and mounted with vectamount. Slides were visualized using a Leica DMLB microscope (Leica Microsystems, Buffalo Grove, IL, USA) equipped with a Leica DFC420 camera and cropped using Adobe Illustrator CS5 software (Adobe, San Jose, CA, USA).

\section{APRIL reporter cell assays}

To measure APRIL signaling, JTF reporter cells, provided as a kind gift by P. Schneider, ${ }^{27}$ were cultured with different APRIL stimuli for $24 \mathrm{~h}$, after which cell death of JTF cells was measured by Dioc6-PI staining as described before. ${ }^{36}$

\section{Flow cytometry and cell viability}

Cell viability was measured by Dioc6-PI staining as described before. ${ }^{36}$ Flow cytometrical staining for APRIL receptors was performed using the TACI-PE (BD Bioscience, San Jose, CA, USA) and BCMA-FITC (Enzo) antibodies as described previously ${ }^{2}$ and stained cells were analyzed on a FACS Canto II (BD). Data was then analyzed using FlowJo 9.9 (FlowJo LLC, Ashland, OR, USA).

\section{Western blot}

Western blotting was performed as described previously, ${ }^{38}$ using the $a$-human APRIL-y1 antibody (Abcam, Cambridge, MA, USA) and $\beta$-actin (Santa Cruz, Dallas, TX, USA) as a loading control. IRDye 680 donkey antirabbit IgG and IRDye 800 donkey anti-goat IgG (Westburg, Leusden, The Netherlands) were used as secondary antibodies.

\section{Real-time polymerase chain reaction (qPCR)}

Total RNA was isolated from paraffin-embedded CLL LN material or from APRIL overexpressing 3T3 cells using the GeneElute Mammalian Total RNA Miniprep kit (Sigma) and CDNA was created by reverse transcriptase reaction according to manufacturer's instructions (Promega, Madison, Wl, USA). APRIL and household gene hypoxanthine phosphoribosyltransferase 1 (HPRT) were amplified using exon-exon boundary overlapping probes (APRIL 5'-CTGCTATAGCGCAGGTGTCTT-3' and 5'-GGAAGGTTCCATGTGGA GAG-3'; HPRT 5'-CCTGGCGTCGTGATTAGTGA-3' and 5'-CGAGCAAGACGTTC AGTCCT-3) in a SYBR green (Life Technologies, Austin, TX, USA) reaction ( 40 cycles of $3 \mathrm{~s}$ at $95^{\circ} \mathrm{C}$ followed by $30 \mathrm{~s}$ at $60^{\circ} \mathrm{C}$ ). The expression of APRIL was then calculated per sample as the difference in Ct values between the APRIL signal and HPRT signal using the formula $1000 \times 2^{-(C t}$ APRIL - Ct HPRT).

\section{Cell proliferation assays}

Cell proliferation was assessed by CFSE cell tracing as described before, ${ }^{2}$ using $200 \mathrm{ng} / \mathrm{ml}$ rhAPRIL (rhA; Peprotech, Rocky Hill, NJ, USA) and other reagents as described before. ${ }^{2}$ Division indices were calculated using FlowJo 9.9 (FlowJo LLC).

\section{Macrophage and NLC experiments}

Monocyte-derived macrophages and NLCs were obtained by differentiating monocytes isolated from healthy donor buffy coats obtained from the central blood bank after obtaining written informed consent. To this end, PBMCs were isolated using ficoll gradient purification (Lucron, Dieren, The Netherlands), after which monocytes were separated from PB lymphocytes using percoll gradient purification (GE healthcare, Milwaukee, WI, USA), both according to manufacturer's instructions. Next, monocytes were incubated to adhere at $37^{\circ} \mathrm{C}$ in $5 \% \mathrm{CO}_{2}$ for $40 \mathrm{~min}$ at a concentration of $0.75 \times 10^{6}$ cells $/ \mathrm{ml}$ in 6 -well plates $(3 \mathrm{ml})$ in IMDM/1\% FBS and washed to remove non-adherent cells. The monocytes were then differentiated towards $\mathrm{M} 1$ macrophages using $10 \mathrm{ng} / \mathrm{ml}$ IFN-Y in IMDM+/+ for $72 \mathrm{~h}$ or to NLCs by differentiating them using CLL cells for 10 days. ${ }^{3}$ After washing the macrophages or NLCs twice, they were pre-incubated for $30 \mathrm{~min}$ with $\mathrm{TACl}-\mathrm{Fc}$ or an equimolar concentration of control lgG (R\&D systems) to obtain a final concentration of $2.5 \mu \mathrm{g} / \mathrm{ml}$ of TACl-Fc. Next, thawed CLL cells were added. After $72 \mathrm{~h}$, cell viability was measured as described before. ${ }^{36}$

\section{Statistical analysis}

One-way analysis of variance (ANOVA) with Tukey post hoc tests (comparing all groups to each other) or Dunnet's post hoc test (comparing all groups to one group) were performed to test for significant differences 
between multiple groups. When applicable, tests were adjusted for repeated measures. A two-way ANOVA with Bonferroni post hoc tests was used when testing for differences between groups with two independent variables. When testing for differences between two groups, a $t$-test was used. $P$-values $<0.05\left(^{*}\right), P<0.01\left(^{* *}\right)$, and $P<0.001\left(^{* * *}\right)$ were considered statistically significant, non-significance is not indicated except in Figures $4 c$ and $d$.

\section{ABBREVIATIONS}

APRIL, a proliferation-inducing ligand; BCMA, B-cell maturation antigen: $\mathrm{CLL}$, chronic lymphocytic leukemia; IFN-Y, interferon gamma; IL, interleukin; JTF, Jurkat-TACl:FAS (reporter cells); LN, lymph node; NF- $K B$, nuclear factor kappa-light-chain-enhancer of activated B cells; PB, peripheral blood; rhA, recombinant human APRIL; SLE, systemic lupus Erythematodes; $\mathrm{TACl}$, transmembrane activator and CAML interactor; TNF, tumor necrosis factor; Tcl-1, T-cell leukemia/lymphoma 1A; ANOVA, one-way analysis of variance; HPRT, hypoxanthine phosphoribosyltransferase 1; CFSE, carboxyfluorescein succinimidyl ester; NLCs, nurse-like cells.

\section{ACKNOWLEDGEMENTS}

We would like to thank Pascal Schneider for providing the JTF reporter cells, Jan-Paul Medema, and Kate Cameron for the TWEPRIL construct and APRIL conditioned medium, and Steven Pals for the CLL LN material. APK is a Dutch Cancer Foundation fellow. This work was supported by the Dutch Cancer Foundation (Dutch Cancer Society Clinical Fellowship UVA 2011-5097).

\section{AUTHOR CONTRIBUTIONS}

MHAvA designed and performed the experiments, analyzed the data and wrote the manuscript; ST and ER designed and performed experiments, and analyzed the data; APK and EE designed the experiments and wrote the manuscript.

\section{COMPETING INTERESTS}

The authors declare no conflict of interest.

\section{REFERENCES}

1 Burger JA. Nurture versus nature: the microenvironment in chronic lymphocytic leukemia. Hematology Am Soc Hematol Educ Program 2011; 2011: 96-103.

2 Pascutti MF, Jak M, Tromp JM, Derks IA, Remmerswaal EB, Thijssen R et al. IL-21 and CD40L signals from autologous $T$ cells can induce antigen-independent proliferation of CLL cells. Blood 2013; 122: 3010-3019.

3 Burger JA, Tsukada N, Burger M, Zvaifler NJ, Dell'Aquila M, Kipps TJ. Blood-derived nurse-like cells protect chronic lymphocytic leukemia B cells from spontaneous apoptosis through stromal cell-derived factor-1. Blood 2000; 96: 2655-2663.

4 Endo T, Nishio M, Enzler T, Cottam HB, Fukuda T, James DF et al. BAFF and APRIL support chronic lymphocytic leukemia B-cell survival through activation of the canonical NF-kappaB pathway. Blood 2007; 109: 703-710.

5 Hendriks J, Planelles L, de Jong-Odding J, Hardenberg G, Pals ST, Hahne M et al. Heparan sulfate proteoglycan binding promotes APRIL-induced tumor cell proliferation. Cell Death Differ 2005; 12: 637-648.

6 Mikhail BL, Bernardetta N, David MH, Bing H, Andras S, Paolo C et al. DCs induce CD40-independent immunoglobulin class switching through BLyS and APRIL. Nat Immunol 2002; 3: 822-829.

7 Yu G, Boone T, Delaney J, Hawkins N, Kelley M, Ramakrishnan M et al. APRIL and TALL-I and receptors BCMA and TACI: system for regulating humoral immunity. Nat Immunol 2000; 1: 252-256.

8 Belnoue E, Pihlgren M, McGaha TL, Tougne C, Rochat A-FF, Bossen C et al. APRIL is critical for plasmablast survival in the bone marrow and poorly expressed by early-life bone marrow stromal cells. Blood 2008; 111: 2755-2764.

9 Craxton A, Magaletti D, Ryan EJ, Clark EA. Macrophage- and dendritic cell-dependent regulation of human B-cell proliferation requires the TNF family ligand BAFF. Blood 2003; 101: 4464-4471.

10 Montserrat $C$, Carolina MB, Bing H, Irene $\mathrm{P}$, Weifeng $\mathrm{X}$, April $\mathrm{C}$ et al. Stromal endothelial cells establish a bidirectional crosstalk with chronic lymphocytic leukemia cells through the TNF-related factors BAFF, APRIL, and CD40L. J Immunol 2012; 188: 6071-6083.
11 Kern C, Cornuel J-FF, Billard C, Tang R, Rouillard D, Stenou V et al. Involvement of BAFF and APRIL in the resistance to apoptosis of B-CLL through an autocrine pathway. Blood 2004; 103: 679-688.

12 Nishio M, Endo T, Tsukada N, Ohata J, Kitada S, Reed JC et al. Nurselike cells express BAFF and APRIL, which can promote survival of chronic lymphocytic leukemia cells via a paracrine pathway distinct from that of SDF-1alpha. Blood 2005; 106: 1012-1020.

13 Maia S, Pelletier M, Ding J, Hsu YM, Sallan SE, Rao SP et al. Aberrant expression of functional BAFF-system receptors by malignant B-cell precursors impacts leukemia cell survival. PLoS One 2011; 6: e20787.

14 Pradet-Balade B, Medema JP, Lopez-Fraga M, Lozano JC, Kolfschoten GM, Picard A et al. An endogenous hybrid mRNA encodes TWE-PRIL, a functional cell surface TWEAK-APRIL fusion protein. EMBO J 2002; 21: 5711-5720.

15 Lopez-Fraga M, Fernandez R, Albar JP, Hahne M. Biologically active APRIL is secreted following intracellular processing in the Golgi apparatus by furin convertase. EMBO Rep 2001; 2: 945-951.

16 Hahne M, Kataoka T, Schroter M, Hofmann K, Irmler M, Bodmer JL et al. APRIL, a new ligand of the tumor necrosis factor family, stimulates tumor cell growth. J Exp Med 1998; 188: 1185-1190.

17 Li YJ, Li ZM, Xia ZJ, Li S, Xia Y, Huang HQ et al. High APRIL but not BAFF serum levels are associated with poor outcome in patients with follicular lymphoma. Ann Hematol 2015; 94: 79-88.

18 Planelles L, Castillo-Gutierrez S, Medema JP, Morales-Luque A, Merle-Beral H, Hahne M. APRIL but not BLyS serum levels are increased in chronic lymphocytic leukemia: prognostic relevance of APRIL for survival. Haematologica 2007; 92: 1284-1285.

19 Lascano V, Guadagnoli M, Schot JG, Luijks DM, Guikema JE, Cameron K et al. Chronic lymphocytic leukemia disease progression is accelerated by APRIL-TACI interaction in the TCL1 transgenic mouse model. Blood 2013; 122: 3960-3963.

20 Lourdes P, Carla EC-P, Gijs H, Salette S, Wilson S, Ruth G-C et al. APRIL promotes B-1 cell-associated neoplasm. Cancer Cell 2004; 6: 399-408.

21 Chiorazzi N, Ferrarini M. Cellular origin(s) of chronic lymphocytic leukemia: cautionary notes and additional considerations and possibilities. Blood 2011; 117: 1781-1791.

22 Hussein G, Hakim O-H, Gandhi D, Bruno R, Brigitte G, Jean-Pierre M. A combination of cytokines rescues highly purified leukemic CLL B-cells from spontaneous apoptosis in vitro. PLoS One 2013; 8: 3.

23 Nupur B, Michaela R, Maiwen CH, Sarah H, Nathan B, Susanne D et al. Loss of cooperativity of secreted CD40L and increased dose-response to IL4 on CLL cell viability correlates with enhanced activation of NF-kB and STAT6. Int J Cancer 2015; 136: 65-73.

24 Schultze JL, Michalak S, Seamon MJ, Dranoff G, Jung K, Daley J et al. CD40-activated human B cells: an alternative source of highly efficient antigen presenting cells to generate autologous antigen-specific $T$ cells for adoptive immunotherapy. J Clin Invest 1997; 100: 2757-2765.

25 Girbl T, Hinterseer E, Grossinger EM, Asslaber D, Oberascher K, Weiss L et al. CD40-mediated activation of chronic lymphocytic leukemia cells promotes their CD44-dependent adhesion to hyaluronan and restricts CCL21-induced motility. Cancer Res 2013; 73: 561-570.

26 Pallasch CP, Schulz A, Kutsch N, Schwamb J, Hagist S, Kashkar $\mathrm{H}$ et al Overexpression of TOSO in CLL is triggered by B-cell receptor signaling and associated with progressive disease. Blood 2008; 112: 4213-4219.

27 Bossen C, Cachero TG, Tardivel A, Ingold K, Willen L, Dobles M et al. TACl, unlike BAFF-R, is solely activated by oligomeric BAFF and APRIL to support survival of activated B cells and plasmablasts. Blood 2008; 111: 1004-1012.

28 Guadagnoli M, Kimberley FC, Phan U, Cameron K, Vink PM, Rodermond $\mathrm{H}$ et al. Development and characterization of APRIL antagonistic monoclonal antibodies for treatment of B-cell lymphomas. Blood 2011; 117: 6856-6865.

29 Napetschnig J, Wu H. Molecular basis of NF-kappaB signaling. Annu Rev Biophys 2013; 42: 443-468.

30 Van Attekum MHA, Terpstra S, Reinen E, Von Lindern M, Slinger E, Eldering E et al. The T-Cell/CLL/Macrophage Triad Shapes a Supportive Tumor Microenvironment in CLL. Abstract 1715 at the Annual meeting of the American Society of Hematology; 4-8 Dec 2015; Orlando, FL, USA. In: Blood 2015; 126 (23).

31 Ansell SM, Witzig TE, Inwards DJ, Porrata LF, Ythier A, Ferrande L et al. Phase clinical study of atacicept in patients with relapsed and refractory B-cell nonHodgkin's lymphoma. Clin Cancer Res. 2008; 14: 1105-1110.

32 Micah JB, Stacey RD, Emanuela C, Raif SG, Shengli X, Kong-Peng L et al. Cutting edge: the dependence of plasma cells and independence of memory $B$ cells on BAFF and APRIL. J Immunol 2008; 180: 3655-3659.

33 Castigli E, Scott S, Dedeoglu F, Bryce P, Jabara H, Bhan AK et al. Impaired IgA class switching in APRIL-deficient mice. Proc Natl Acad Sci USA 2004; 101: 3903-3908.

34 Seshasayee D, Valdez P, Yan M, Dixit VM, Tumas D, Grewal IS. Loss of TACI causes fatal lymphoproliferation and autoimmunity, establishing $\mathrm{TACl}$ as an inhibitory BLyS receptor. Immunity 2003; 18: 279-288. 
$35 \mathrm{Xu} \mathrm{S}$, Lam KP. B-cell maturation protein, which binds the tumor necrosis factor family members BAFF and APRIL, is dispensable for humoral immune responses. Mol Cell Biol 2001; 21: 4067-4074.

36 Tromp JM, Tonino SH, Elias JA, Jaspers A, Luijks DM, Kater AP et al. Dichotomy in NF-kappaB signaling and chemoresistance in immunoglobulin variable heavychain-mutated versus unmutated CLL cells upon CD40/TLR9 triggering. Oncogene 2010; 29: 5071-5082.

37 Hallaert DY, Jaspers A, van Noesel CJ, van Oers MH, Kater AP, Eldering E. c-Abl kinase inhibitors overcome CD40-mediated drug resistance in CLL: implications for therapeutic targeting of chemoresistant niches. Blood 2008; 112: 5141-5149.
38 Mackus WJ, Lens SM, Medema RH, Kwakkenbos MJ, Evers LM, Oers MH et al. Prevention of $\mathrm{B}$ cell antigen receptor-induced apoptosis by ligation of CD40 occurs downstream of cell cycle regulation. Int Immunol 2002; 14: 973-982.

\section{cc) (i)} article are included in the article's Creative Commons license, unless indicated otherwise in the credit line; if the material is not included under the Creative Commons license, users will need to obtain permission from the license holder to reproduce the material. To view a copy of this license, visit http://creativecommons.org/licenses/by/4.0/

Supplemental Information accompanies the paper on the Cell Death and Discovery website (http://www.nature.com/cddiscovery). 